\title{
Local Detectors in Preventive Lightning Protection
}

\author{
Attila Gulyás ${ }^{*}, 1$, Jakke Mäkelä",2, Bálint Németh ${ }^{1}$, Vernon Cooray ${ }^{3}$, István Kiss ${ }^{1}$ and István Berta ${ }^{1}$ \\ ${ }^{I}$ Budapest, University of Technology and Economics, Department of Power Engineering, Hungary \\ ${ }^{2}$ Nokia Devices, PO Box 86, 24101 Salo, Finland \\ ${ }^{3}$ Angström Laboratory, University of Uppsala, Sweden
}

\begin{abstract}
In lightning protection theory the preventive lightning protection (PLP) deals with using the forecasting tools for protection, combining them with special preventive actions. In zonal preventive lightning protection (ZPLP) location inaccuracies in the forecasting tools are assumed to be negligible. This approach is justified in the case of high-end measurement devices such as lightning detection networks. However, as the electronics industry is able to miniaturize sensors more and more, a new class of warning devices, is emerging aimed at very low-cost real-time warnings of lightning activity near individual structures. These local sensors in general have the characteristic that their inaccuracy grows as the distance to the flash grows. Also some sensors are capable of signalling the development of a thunderstorm cell before lightning activity starts. Such inhomogenity is not covered in traditional PLP theory. This paper deals with the theoretical introduction of such local detectors into the theory of preventive lightning protection.
\end{abstract}

Keywords: Lightning protection, preventive lightning protection, lightning detection.

\section{INTRODUCTION}

Preventive lightning protection (PLP) is a dynamic protection method, which uses the available lightning hazard forecasting tools to trigger preventive actions [1]. The preventive actions are active while the lightning hazard exists and are suspended once it's not present anymore. So unlike primary and secondary protection methods, preventive lightning protection does not use static devices installed to the object to be protected. Due to this behaviour the object to be protected is not continuously protected, but only when the preventive actions are in effect. This may result in cases, when the preventive action is not executed in time, so the accuracy of the forecasting tool is vital in this method.

Nowadays the lightning detection networks provide ample information about the lightning activity and thus are good tools for preventive lightning protection. On the other hand acquiring such detailed, real-time information is quite costly, so in many situations the standalone low cost detectors are to be used instead.

These detectors have special properties compared to the lightning detection networks. In this paper the local detectors are introduced into preventive lightning protection. These standalone devices can be used in each type preventive lightning protection (ZPLP, HRPLP and FPLP - explained in later sections) using results on local detector performance [24]. This paper shows how the local detectors may be used with each type based on a simulation, and simple comparison of the performance of different PLP types is also given.

\footnotetext{
*Address correspondence to these authors at the Budapest, University of Technology and Economics, Department of Power Engineering, Hungary; Tel: +36 1463-2904; Fax: +36 1 463-3600;

E-mail: gulyas.attila@vet.bme.hu and Nokia Devices, PO Box 86, 24101

Salo, Finland; E-mail: Jakke.Makela@nokia.com
}

\section{CHARACTERISTICS AND PERFORMANCE OF LOCAL DETECTORS}

Lightning detectors are capable of sensing the electromagnetic field caused by lightning. A high accuracy is possible by using multiple wideband detectors with high time resolution, ideally with each station capable of distancefinding. At such systems, location accuracies can be $500 \mathrm{~m}$ or better [5]. With this level of accuracy and speed of response, these warning systems can be used as a "golden standard" in the preventive lightning protection theory.

Standalone local detectors on the other hand offer a lowcost solution compared to lightning detection systems, with lower regarding strike point location accuracy. The local detectors are also capable of sensing either the electrical field caused by lightning activity [2-4] or the increasing field at ground level caused by the developing electrical activity [68]. The former category includes the narrow band (HF based) sensors while the latter includes the electric field mills. In this paper, we focus on the devices described in [2], since they are the most fully described in the public literature. However, the same basic technique is valid for all local detectors, and indeed for any lightning detection network. This is because even CG lightning has large horizontal extent [9], and therefore the location of the ground strike can be kilometers away from the in-cloud origin of the flash. The position of a ground flash therefore provides only an approximate guide to the probable location of the next ground flash, and does not predict risk very accurately.

In the technique proposed by [2-4], the intensity of the radiation from a lightning discharge (at some particular frequency) is used to estimate the distance to the thunderstorm cell. The ranging accuracy of individual lightning discharges is very poor because of flash-to-flash variations as well as variations in ground attenuation. Thus, 
the ranging accuracy of such devices decreases as a function of distance.

Because of this fundamental limitation a zonal approach was proposed for using local detectors $[2,4]$. The purpose of this zonal approach is twofold. First, since local detectors are to be used for giving an advance warning it is crucial to scale the devices sensitivity (and reliability) to provide accurate warning. Second, these devices may be tuned to some extent and therefore the proposed zonal approach may be used to set right sensitivity values depending on the application - the required timing of warning.

\section{Three zones were defined in [2]:}

Zone 1 (danger zone): ranging within this zone does not in fact have to be highly accurate; whenever a flash is in this range, the user is endangered. Thus the user shall be notified of the presence of the thunderstorm cell in this zone, as it poses a risk of direct or indirect lightning strike ${ }^{1}$.

Zone 2 (tracking zone): this is the zone in which the ranging accuracy has to be the highest; in particular, transitions from Zone 2 to Zone 1 must be detected accurately and rapidly. The user may be notified, that there is a storm further away (which does not pose a direct threat), but warning is not given in this case, as there is no risk of damage. As ground characteristics affect ranging accuracy, the maximum distance for Zone 2 is generally about $20 \mathrm{~km}$.

Zone 3 (monitoring zone): this zone corresponds to the maximum confident ranging distance. Within this range, lightning flashes are likely to be observed, but their ranging accuracy becomes poor. It is argued in [4] that in this zone, it is sufficient to warn of the existence of lightning flashes, without even attempting accurate ranging. It is also argued that past the upper range (about $30 \mathrm{~km}$ ) flashes should be ignored altogether.

This zoning approach enables to use the local detectors as tools of forecasting, and makes them compatible with the approach of preventive lightning protection. The zones may be explicitly mapped to any PLP solution - see section 3 for a more thorough description on the zones used in PLP and how zones 1-2-3 are mapped accordingly.

The performance of local lightning detectors is illustrated for this paper by a simple simulation which, while not fully descriptive of a real thunderstorm, allows the introduction of the usage of these local detectors in various realizations of PLP (ZPLP, HRPLP and FPLP - discussed in details in Section 3, 4 and 5) and its analysis.

The simulated storm is assumed to consist of CG flashes whose ground attachment points steadily approach the observation point. The first flash occurs at a distance of 30 $\mathrm{km}$. After that, three flashes occur per minute (corresponding to a medium-intensity storm). The storm is then assumed to approach the observation point at the speed of $30 \mathrm{~km} / \mathrm{h}$. Thus, the flashes are $20 \mathrm{~s}$ apart and are separated by exactly $333 \mathrm{~m}$. When the cell reaches within $1 \mathrm{~km}$ of the observation point, it is assumed to recede at the same rate.

${ }^{1}$ The authors of [2] used the "30-30 rule" found in [10] when describing the zonal structure. This rule is very simple and easily adaptable in some situations. PLP however assumes a scientifically more valid approach. This is one of the reasons why the zonal approach of Mäkelä is slightly refined to be compatible with PLP.
The next part of the simulation is determining what ground flash distance the sensor returns (instead of the steady closing strike points $333 \mathrm{~m}$ from each other). In order to produce the series of simulated values we have to consider the characteristics of the sensor. The sensor is assumed to be isotropic, and all propagation effects are ignored. The energy is assumed to have a simple relationship observed by [2], namely that the dimensionless intensity I drops as $I(R)=I_{0} *\left(R_{0} / R\right)^{k}$ where $\mathrm{I}_{0}$ is the flash intensity at reference distance of $R_{0} \mathrm{~km}, \mathrm{R}$ is the distance to the flash in $\mathrm{km}$, and $\mathrm{k}$ is an empirically determined constant with value $k=3.1$. The equation gives an exact range only if all flashes have the same source intensity. However, according to the source intensities are log-normally distributed, with parameters $\mu=1$ and $\sigma^{*}=0.34$. Thus any individual flash at distance $R_{\text {real }}$ will be ranged to a distance:

$R_{x}=R_{\text {real }}\left(I_{x} / I_{\text {median }}\right)^{-1 / k}$

Given the simulated distance values obtained from the sensor a basic test on accuracy may be performed. That means checking when the transitions between the given zones were observed compared to when they should have been observed (see section 5 for detailed results of this analysis). Due to the variance of the data certain smoothing is to be used to be able to determine the distance to the ground flash.

A very simple running average of [2] is used to estimate this distance. A buffer of 30 measured distances is kept, and the four closest flashes are averaged to give an estimate for the closest edge.

One sample run is shown in Fig. (1). The real distance to the ground attachment is shown by the diagonal red lines, and the zone ( 1 and 2) boundaries are marked with the horizontal (red) lines at $20 \mathrm{~km}$ and $10 \mathrm{~km}$ respectively.

The dots are the simulated measured distances. The solid blue line shows the distance as estimated by the floatingaverage method. It is seen that the approach is modelled reasonably well after the first 30 flashes have been observed. There is some lead time, i.e. the sensor places the storm slightly closer than the real distance. In particular, when the storm begins to recede, the floating average shows the storm to be receding too slowly. This is a known weakness in the method.

Under these idealized conditions, then, the warning (given on entering Zone 1) begins in median 3 minutes early (range 0-4 minutes) and ends in median 4 minutes too late (range 2-7 minutes). Depending on cost efficiency concerns these errors are 'acceptable'.

In the following sections various PLP methods are extended with the local detectors. In section 5 a comparison of different methods (PLP and also this averaging method presented in this section) is given.

\section{ZONAL PREVENTIVE LIGHTNING PROTECTION AND LOCAL DETECTORS}

There are three possible solutions of preventive lightning protection. They are characterized (and named) by the forecasting method used in the solution. In this section the theoretical background of ZPLP is discussed first, and then the methodology of using local detectors is shown. 


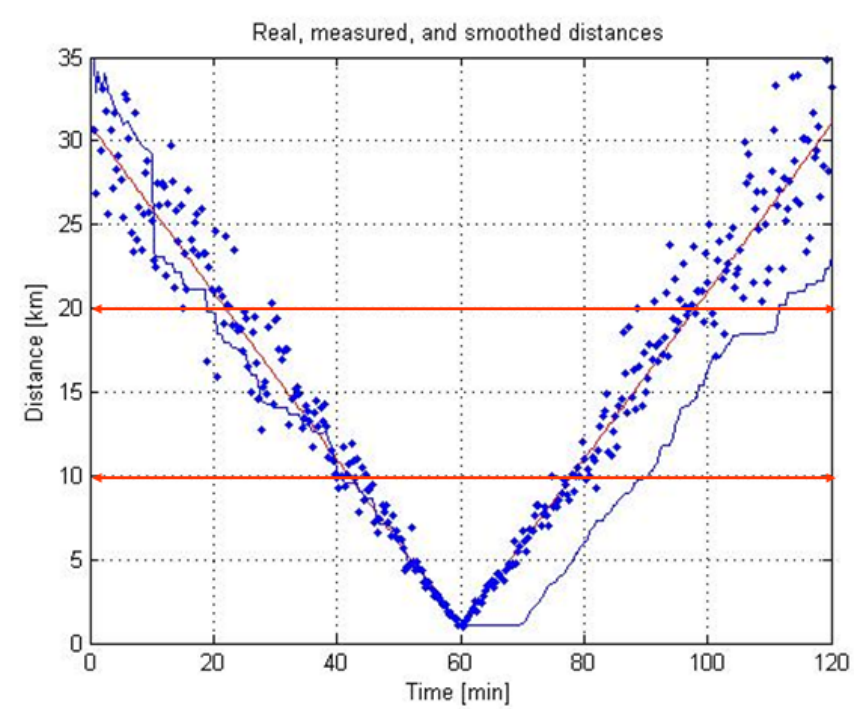

Fig. (1). Simulated approach of a storm. Red line shows location of flashes, blue dots are the measured distances given that there are flash-by-flash variations. The blue solid line is the floating average of the four closest flashes among the 30 preceding flashes.

Zonal Preventive Lightning Protection (ZPLP) uses two zones to provide an advance warning to execute the preventive action in time.

In Fig. (2), the zones used in ZPLP are shown. The zone denoted in red around the object to be protected is the Danger Zone (DZ); the green zone is the so-called Warning Zone (WZ) $[11,12]$.

The Danger Zone denotes the area around the object to be protected where the presence of an active thunderstorm cell means lightning hazard. Lightning hazard means, that the object to be protected is endangered (exposed to direct/indirect strikes) by an active thunderstorm cell. The thunderstorm cell is denoted active once it is electrically active (IC and/or CC discharges are observed). After the initial discharges CG lightning is produced. So when observing IC or CC activity the possibility of CG strokes may not be neglected.

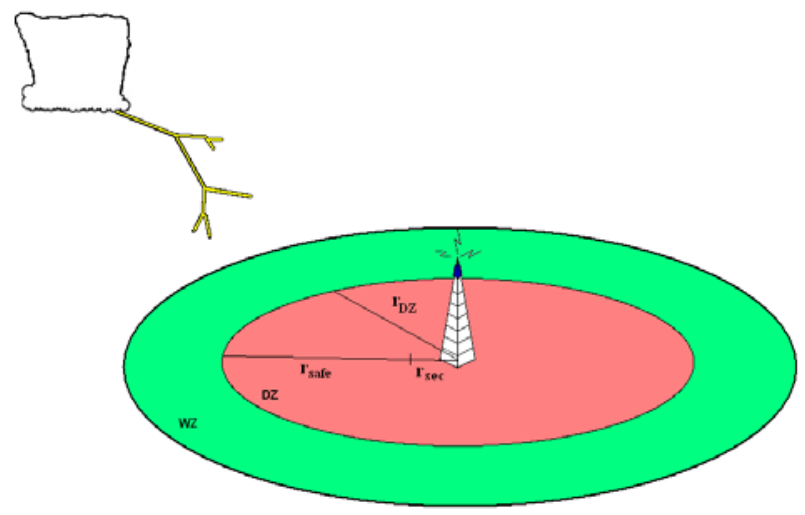

Fig. (2). Zonal preventive lightning protection.

At the time the active thunderstorm cell enters the DZ, the preventive action has to be executed already; the object to be protected has to be already in the protected state. When the object to be protected is in the protected state, the risk of damage due to lightning strike - either direct or indirect - is decreased.

The size of the DZ is determined by assumptions of the distance from where direct lightning strikes may occur (how far a lightning may strike from a thunderstorm cell), but the secondary effects of an indirect lightning strike are also to be taken into account.

$r_{D Z}=r_{\mathrm{sec}}+r_{\text {safe }}$

In (2) $r_{s e c}$ denotes the distance where the strike produces secondary effects, and $r_{\text {safe }}$ denotes the safety distance - the radius where a lightning discharge may reach from the thunderstorm cell. There are several sources for this radius mentioning either $2,5,10 \mathrm{~km}$ or even more [13]. The DZ is used in each type of PLP.

To assure the timely execution of the preventive action, a Warning Zone is used. The WZ denotes the area around the DZ where the presence of the thunderstorm cell shall trigger the alarm to start the execution of the preventive action. The size of this zone depends on the time requirement of the preventive action execution. Ideally once a thunderstorm cell enters the WZ and an alarm is given, the object to be protected will already be protected once the thunderstorm cell reaches the border of the DZ. For the complete description of the event space approach and PLP theory see [12].

$r_{W Z}=r_{D Z}+t_{a c t} v_{s t o r m}$

Expression (3) gives the radius of the WZ calculated from the object to be protected. The first term denotes the size of the DZ explicitly. The second term corresponds to the distance depending on average thunderstorm speed and the time requirement of the preventive action. The 'average thunderstorm speed' in this case is a guideline. Depending on user requirements the size of the WZ may be chosen to assure the timely execution of the preventive actions even in case of high wind speeds (and thus higher thunderstorm velocities). This may result in a higher number of unnecessary alarms, but reduces the probability of getting a late alarm [14].

However ZPLP is only one approach to forecasting in preventive lightning protection and simply with the calculation of zone radii, the protection can be planned, the event space may be calculated and the accordance with the standard may be checked.

Two other types of PLP are introduced in later sections in the scope of local detectors. The first type uses real-time monitoring of thunderstorm cells is denoted as High Reliability Preventive Lightning Protection (HRPLP, see [15]) may also be applied when using local detectors. This type is discussed only briefly, as it is fundamentally similar to fuzzy preventive lightning protection (FPLP $[19,20]$ introduced in details in section 4), but does not use a fuzzy inference system. Both HRPLP and FPLP methods use realtime lightning data constantly monitoring the propagation direction and velocity of thunderstorm cells.

When using local detectors in ZPLP, planning is done easily when using lightning detection networks. Local detectors may also be used in ZPLP, but according to their performance, they have to be handled differently. 


\subsection{Including Local Detectors into ZPLP Theory}

Generally the local detectors are to be used either exclusively, or as a supplement for lightning detection networks. The former solution is discussed here only. When used as a supplement, usually field mills are applied, since the lightning detection networks are capable of detecting electrical discharges only, but not the preceding events. Field mills are capable of measuring the electric field in an area, which is a good predictor of thunderstorm cell activity [7], and there are also other tools of measuring electrical activity [16].

Using local detectors as stand-alone devices for detection yields a low cost, yet not necessarily less accurate solution. The local detectors may be used two ways. A local detector may be explicitly placed at the object to be protected or it is also possible to build a 'perimeter' of local detectors [17] to provide a 'map' of electrical activity around the object to be protected (the former is discussed here only).

In the basic ZPLP theory, it is implicitly assumed that the detection system is "perfect", in the sense that lightning activity $(\mathrm{CC} / \mathrm{CG} / \mathrm{IC})$ in the $\mathrm{WZ}$ will be detected accurately and in real time. With high-quality lightning detection networks, this idealization may be acceptable.

Whether such a perfect system exists is an open question. Even with high-quality lightning detection networks, the large horizontal extent of most flashes [9] means that the location of the ground attachment point would be only a rough approximation of the real location of the thunderstorm. When single-station detectors of the type described by [2] are used, the detection system is fundamentally not perfect, and needs to be analyzed by the methods in this paper. It is left for further study to determine which detection systems, if any, can be considered perfect.

The fundamental question when incorporating local detectors into the PLP theory is how to account for the fact that the ranging accuracy is not constant; and ranging can only be done statistically.

As shown in section 2 and [2], the local detectors are best described by a zonal approach as well. Zones 1, 2 and 3 can directly be mapped to PLP with only a few restrictions.

First, the size of Zone 1 should be smaller or equal than the DZ radius (for whichever forecasting method). This is crucial to accurately sense when the thunderstorm cell enters the DZ. Second, the border of the WZ should be within Zone 2 as this provides an accurate signal of a thunderstorm cell entering the WZ - thus an accurate alarm to start the preventive action execution.

The application of such detectors in ZPLP does not yield any changes in the theoretical calculations (see [12]), but its performance measures - the bounds for Zone 2 and Zone 3 are required to plan a reliable solution. Also multiple standalone sensors may be used in ZPLP.

\subsection{Alarming Conditions for ZPLP Using Local Detectors}

The input from a local detector is an intensity and as such an estimate to the distance of the thunderstorm cell. The characteristics described in section 2 show that the farther the thunderstorm cloud is, the less accurate the distance approximation gets. In ZPLP the accurate measurement of the thunderstorm cell distance is crucial to monitor crossing zone boundaries. The input from the local detector is directly used as a signal for alarming.

Fig. (3) shows how misleading the inaccuracies may be when making the alarming decisions. When using distance data obtained from a stand-alone detector we have to be aware of the relatively big variance in the data. This may be decreased by using averaging methods. One possible solution shown in Section 2 is the floating averaging. To further decrease the errors of this method we propose the use of weighted floating averaging.

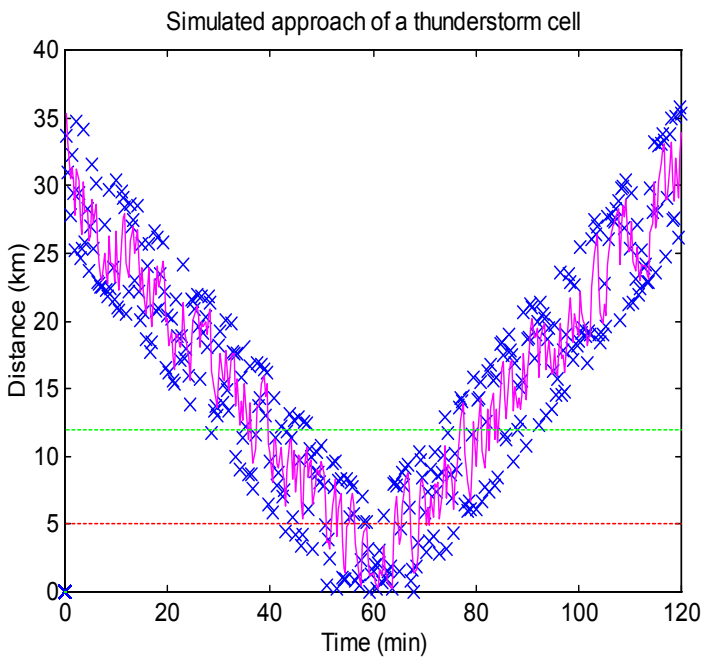

Fig. (3). The characteristics of a local detector, DZ and WZ marked red and green respectively.

Supposing that a result was obtained at $t_{i}$ and the previous calculation was done in $t_{i-1}$ the floating weighted average is calculated the following way.

$a\left(t_{i}\right)=\frac{1}{2}\left(a\left(t_{i-1}\right)+d\left(t_{i}\right)\right)$

In this expression $a\left(t_{i}\right)$ means the floating weighted average in $t_{i}$ and $d\left(t_{i}\right)$ means the distance data gotten from the sensor at $t_{i}$. This means that previous samples have a decreasing weight. Practically when written with a noniterative expression this weighting results in the following calculation

$a\left(t_{i}\right)=\frac{1}{2} d\left(t_{i}\right)+\frac{1}{4} d\left(t_{i-1}\right)+\frac{1}{8} d\left(t_{i-2}\right)+\ldots+\frac{1}{2^{n+1}} d\left(t_{i-n}\right)$

In the figure above the blue crosses represent actually measured distance data from the detector and the magenta line shows the averages. DZ and WZ borders are shown in red and green (horizontal, $5 / 12 \mathrm{~km}$ away).

Concerning protection efficiency it's crucial to get a timely alarm. It is possible, that an active cell already in the WZ may be detected as one still outside the WZ, thus the alarm is not given in time. To account for this inaccuracy, the size of the WZ shall be determined taking into account this inaccuracy as well. Thus in case of a local detector with a given standard deviation characteristics $\operatorname{std}(r)$ the WZ radius is to be calculated using the following expression: 


$$
r_{W Z}=r_{D Z}+t_{a c t} v_{s t o r m}+\operatorname{std}\left(r_{D Z}+t_{a c t} v_{s t o r m}\right)
$$

It means that once we plan our WZ with an ideal model of a detector, the worst case inaccuracy is to be added to this value to provide timely alarm in any case. The DZ does not have such functionality (no action is taken upon entry, the preventive action should already be in effect) - it has a fundamental meaning instead as written earlier -, so it does not have to be modified. Also since it's much narrower, the accuracy of a local detector is significantly better.

In terms of cost efficiency this modification means a decrease - depending on the original WZ, sometimes a significant decrease -, but if it is required to decrease the risk to acceptable levels, this has to be applied. Using multistaged preventive actions may improve cost efficiency (resulting in using multiple WZs), but it's not in the scope of this paper $[12,18]$.

\section{FUZZY BASED PREVENTIVE LIGHTNING PROTECTION (FPLP) AND LOCAL DETECTORS}

Application of fuzzy inference systems (FIS) in preventive lightning protection was presented in several papers before, e.g. in $[19,20]$. The basis of the application of such a solution for preventive lightning protection was the evaluation of data of lightning detection systems, meteorological radars, etc, real-time data of thunderstorm cells.

In FPLP a DZ mentioned earlier is used in an identical function. Instead of the WZ however, the following initial parameters are taken into account:

- $\quad$ the velocity of the thunderstorm cell $(\mathrm{v}[\mathrm{km} / \mathrm{h}])$

- distance between the thunderstorm and the area to be protected $(\mathrm{r}[\mathrm{km}])$

- direction of the movement of thunderstorm cell: the angle between the line drawn from the centre of thunderstorm cell to the object to be protected and the actual velocity vector ( $\alpha[$ degree $])$.

- $\quad$ size of thunderstorm: diameter of simplified cloud model $(\mathrm{D}[\mathrm{km}])$ or length of a front

- $\quad$ intensity of lightning activity in the thunderstorm cell ([flashes / min])
Using all these data in a Fuzzy Inference System [19] results in an overcomplicated rulebase, therefore it is useful to apply such parameters that are 'pre-processed versions' of the previous ones. As a result of pre-processing, two parameters remain as inputs of a FIS:

- $\quad$ probability, that the thunderstorm enters the DZ, thus reaches the object to be protected

- relative time $t_{r}=\left(t_{\text {reach }}-t_{\text {act }}\right) / t_{\text {act }}$ where $t_{\text {reach }}$ is the estimated time left before the thunderstorm arrives, while $t_{a c t}$ denotes the time necessary to execute the preventive action. This estimation, $t_{a c t}$ is a value modified by the time factor $f_{t}$ (defined below in the fourth paragraph).

Calculation of the probability parameter is quite complicated. Some background of its possible calculation methods can be found in $[12,15]$.

The next step is the fuzzy classification of data. For that purpose, fuzzy sets must be defined by their membership functions. The membership functions are dimensionless, their value being between 0 and 1. It shows the 'degree' of the value belonging to a given fuzzy set. The names of the fuzzy sets are indicated in Figs. (4, 5, surely not ... surely yes and too late...too early). Thus the input quantities are 'fuzzified' (classified to fuzzy sets, actually simplifying the input quantity into ordinal values rather than numerical ones).

Selection of output quantities in our case is a bit more complicated task. Such parameters are necessary that clearly indicate, which scenario is available to execute and when. For that purpose the output parameters provided by FIS systems are

$f_{t}$ : time factor. We suppose that the average time $\left(t_{a v}\right)$ of the preventive action execution is known. Certain circumstances may influence this time, so the actual time value may be obtained by multiplying by $f_{t}$.

$f_{n}$ : necessity factor, a number between 1 and 0 . When its value reaches 1 , the preventive action must be started immediately.

Figs. $(6,7)$ show the membership functions (fuzzification) of the output quantities given to the user. Thus in the end the FIS system outputs one value for each factor: low, average

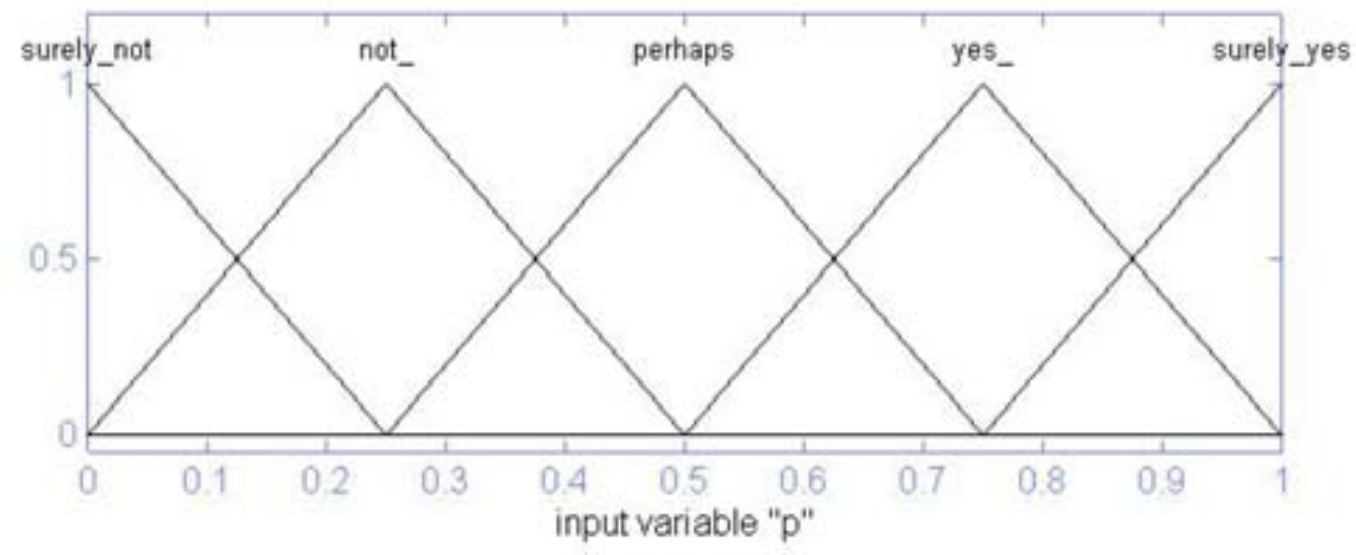

Fig. (4). A membership function (input: probability that the thunderstorm cell enters the DZ). 


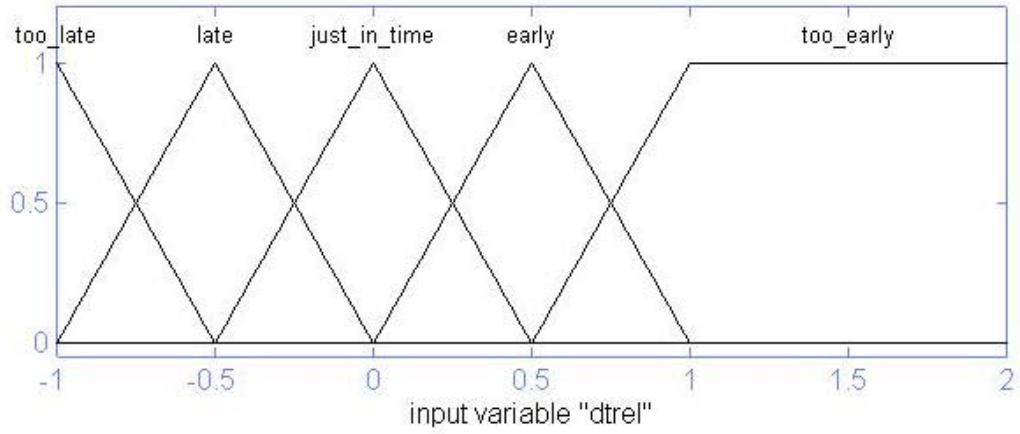

Fig. (5). A membership function (input: time that the thunderstorm cell reaches the DZ).

or high for the time factor, and very_low...very_high for the necessity factor.

As a final step of the analysis, a necessity value and a time value have to be determined. In our analysis using the simulated data the Centre of Gravity method (COG) was used for this purpose [21].

In some cases the input parameters of the inference system are obtained from the change of the distance between the edge of a thunderstorm cell and the DZ. The information can be obtained from lightning detection systems or local (even standalone) detectors.

When using standalone detectors introduced in section one, the information obtained is the distance of the active thunderstorm cell. In this case the input parameters of the FIS are determined from distance data (including the change of distance as a function of time) [20]. That results in a relatively simple FIS solution.

In the case study found in the next section our goal is to determine the different level of the necessity factor at given times. Thus it may be determined when an alarm should be given based on the input data from the standalone sensor.

\section{A COMPARISON OF METHODS USING SIMU- LATION RESULTS}

As the earlier sections show there are multiple ways to use the local detectors in PLP. In this section we'll introduce a short comparison which is based on using 200 Monte Carlo simulated thunderstorms. In our simulation we generated a time series of the distance of the thunderstorm cell which passes above the local detector. Using (1) a measurement data was generated from the time series 200 times. The

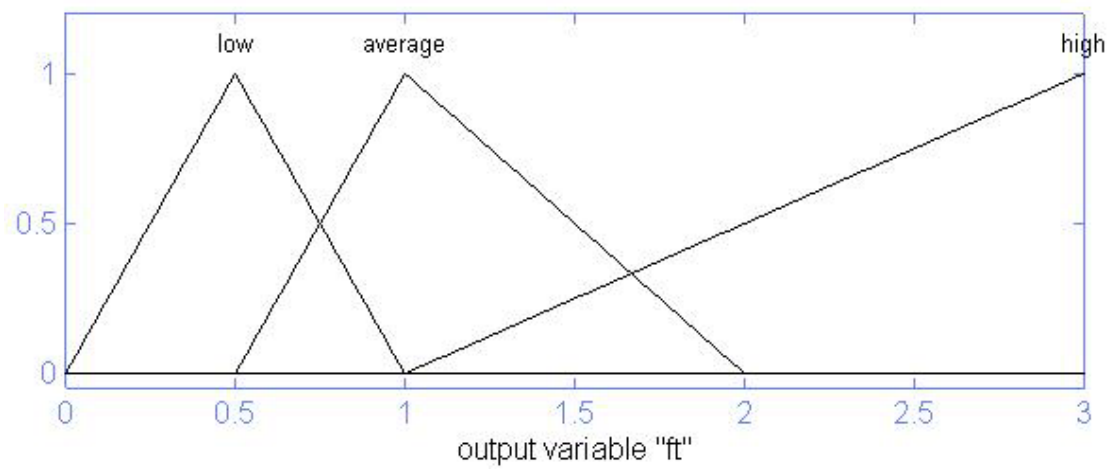

Fig. (6). the time factor as an output variable.

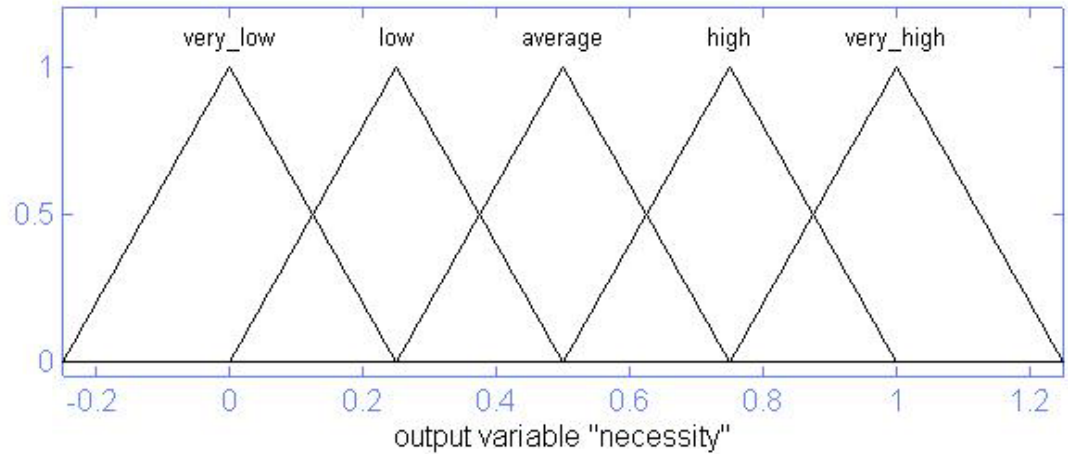

Fig. (7). the necessity factor as an output variable. 
thunderstorm cell simulated was propagating with a constant speed of $30 \mathrm{~km} / \mathrm{h}$ towards the object to be protected, and passes it. The measured distance with the sensor was simulated and used.

First a statistical analysis is shown using the zonal approach (as described in Section 2) for describing sensor performance, then the performance of ZPLP, HRPLP and FPLP is described (following the principles described in Section 3 and 4).

\subsection{Performance of the Stand-Alone Detectors}

The main parameters of interest are the errors in determining the passing of the storm through the Zone boundaries (Zone 2 and Zone 1 boundaries). Fig. (8) and Fig. (9) shows the results of 200 Monte Carlo simulations. In Fig. (8) the panels on the left show the distribution error in determining the Zone $2 \& \# 8211$; the tracking zone \&\#8211; crossing. The top panel shows the lead time when the first flash to apparently cross the zone is taken as the crossing moment. In this case, the sensor systematically warns up to 10 minutes too early, with a median error of 7 minutes. Using the floating average decreases the lead time to a median of 3 minutes.

The more critical Zone 1 (DZ in PLP terms) errors are in the right panel. If a single flash is used to trigger the crossing alarm, the median lead-in error is 3 minutes. In just $2 \%$ of the cases, the lead time is negative, i.e. the sensor produces a late alarm (however, by a time of less than 1 minute). When the floating average is used, the median error is only 2 minutes, but in some $5 \%$ of the cases a late alarm is produced, again only by less than 1 minute.

The left panels show the time using the first flash measured to be inside the zone, while the right panel shows the time the floating average crosses the zone. In almost all
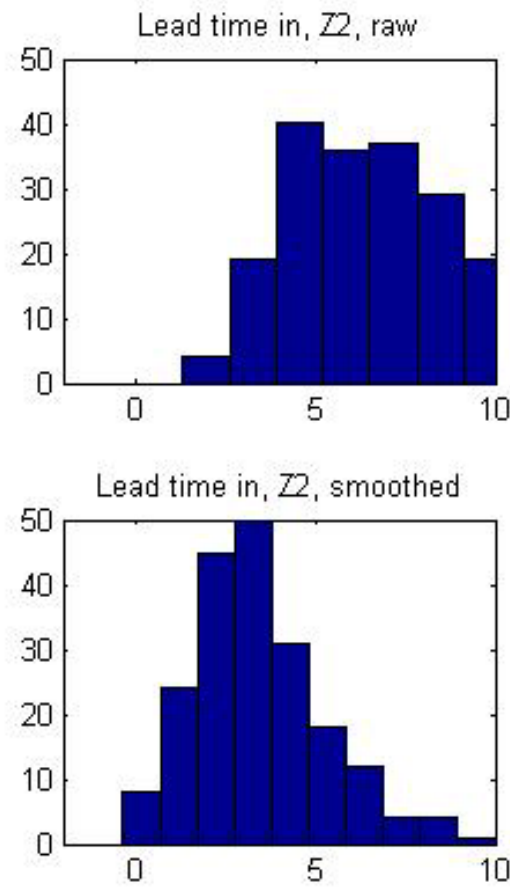

cases, the warning is given slightly before the storm actually reaches the zone.

As Fig. (9) shows, the delay time (time lag between the time the storm leaves the zone and the time when it is observed to do so) is considerably larger. For Zone 2, the median delay for the floating average is 4 minutes, and also for Zone 1 (DZ).

\subsection{Using a ZPLP Solution}

If a standalone device is used in ZPLP then its performance is crucial. Here we assumed that the $\mathrm{WZ}$ radius is smaller than the tracking distance - Zone 2 - as it is a prerequisite to use such a detector.

Using the results of the same simulation, the performance of such a sensor in ZPLP was investigated. The WZ according to (6) - was set to $12 \mathrm{~km}$ with a DZ set to $5 \mathrm{~km}$. The most important question in this case is if the time between the alarm and the cell entering the DZ is sufficient to fully execute the preventive action (given that its execution immediately started after the alarm was given). If it is not, then we denote this case as a late alarm, and the object to be protected was endangered by lightning.

Thus the performance measure to be used is the probability that such a late alarm happens, and a good solution minimizes this probability. Giving an immediate alarm upon the entry into the WZ based on the Monte Carlo simulation gives the following results.

In case of a preventive actions having a time requirement less than 10 minutes this solution is adequate; the probability of late alarms stay below 0.1 .

It is also important to evaluate the ZPLP solution for cells with different propagation speed. Since the WZ size is constant, at higher propagation speed there is less time to execute the preventive action.
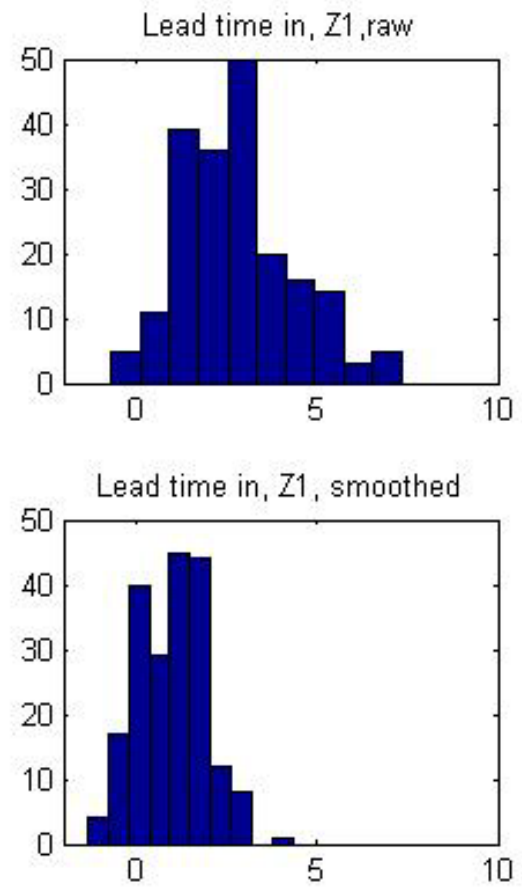

Fig. (8). The effect of the flash-by-flash variations on errors in the measured approach times to DZ and Zone 2 (y axis showing the frequency in the simulations, $\mathrm{x}$ axis showing the actual parameter). 

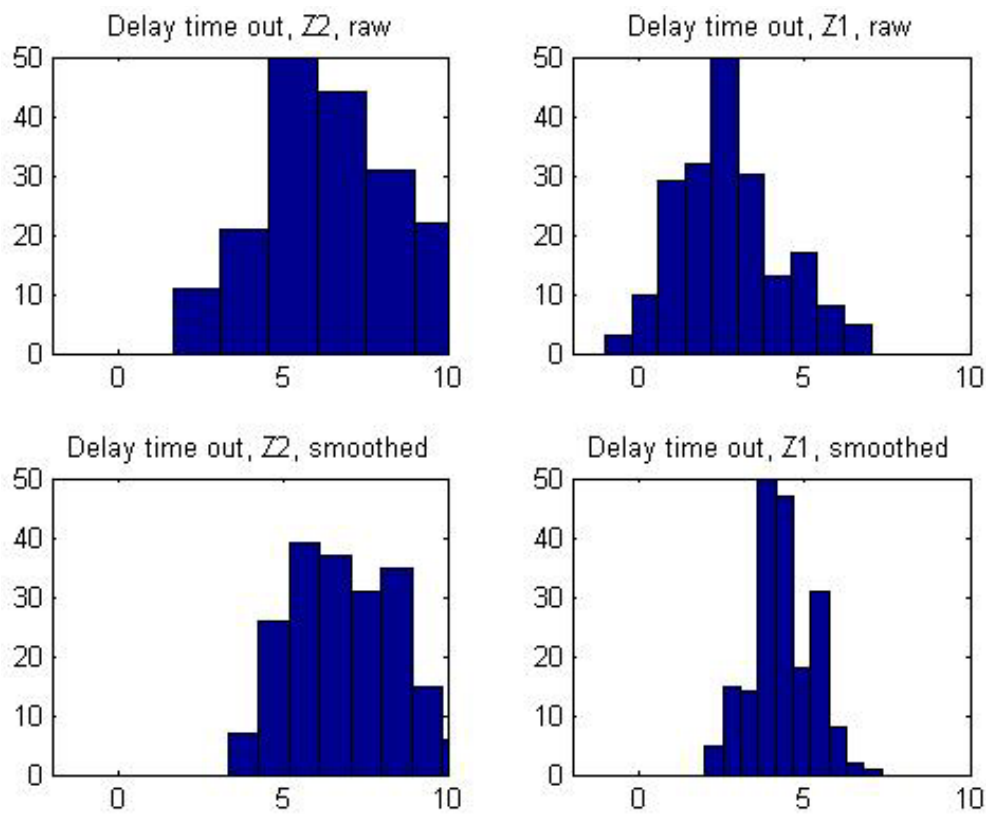

Fig. (9). Effect of the flash-by-flash variations on errors in the measured departure times from the Zone 1 (DZ) and Zone 2 (outer bound for the WZ). The left panels show the time using the last flash measured to be inside the zone, while the right panel shows the time the floating average crosses the zone ( $\mathrm{y}$ axis showing the frequency in the simulations, $\mathrm{x}$ axis showing the actual parameter). In almost all cases, the warning is given several minutes after the storm has passed the zone.

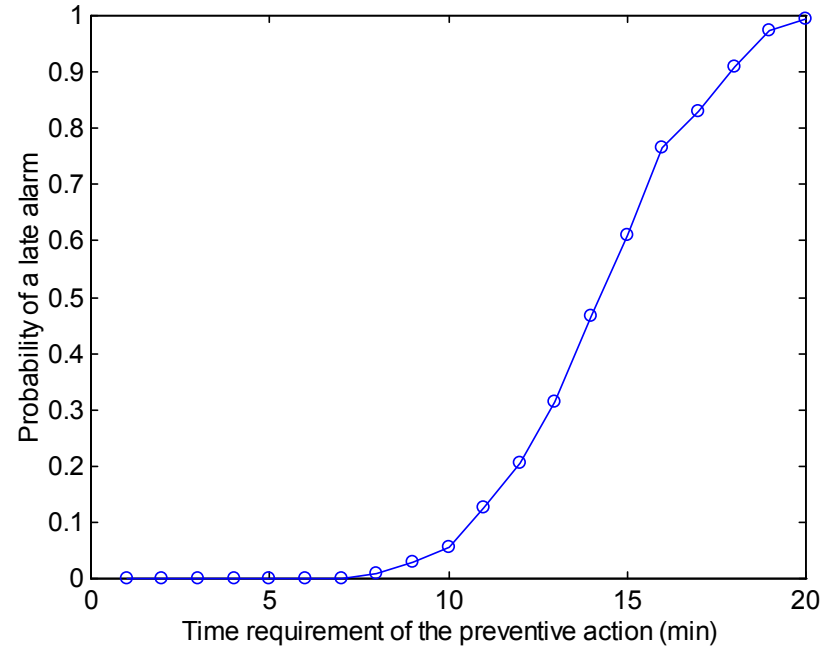

Fig. (10). Late alarms versus time requirements.

Fig. (11) shows the maximal thunderstorm cell velocities at which the current solution provides a timely alarm. As seen the current arrangement of WZ $(12 \mathrm{~km})$ and DZ $(5 \mathrm{~km})$ provides adequate solution if the speed of the thunderstorm cell is below $42 \mathrm{~km} / \mathrm{h}$.

In case of shorter action execution times this solution provides protection against thunderstorm cells with much higher velocity as well. Given a fixed execution time a stricter $v_{\text {storm }}$ parameter may be chosen when calculating the WZ size (see (6)) - this would also bring more cost effective solutions.

\subsection{Using a FPLP or a HRPLP Solution}

Application of fuzzy logic in this case is based on HRPLP. In HRPLP a critical distance $\left(d_{c r i t}\right)$ is determined instead of using a fixed WZ - note that the DZ is still used. The critical distance is changing dynamically taking into consideration $v_{\text {storm }}$ similarly as it is written in (3) -, and the propagation direction (in this case only a velocity value may be calculated of course). For a more thorough analysis on HRPLP see [15]. Due to that similarity these methods are shown together in this section.

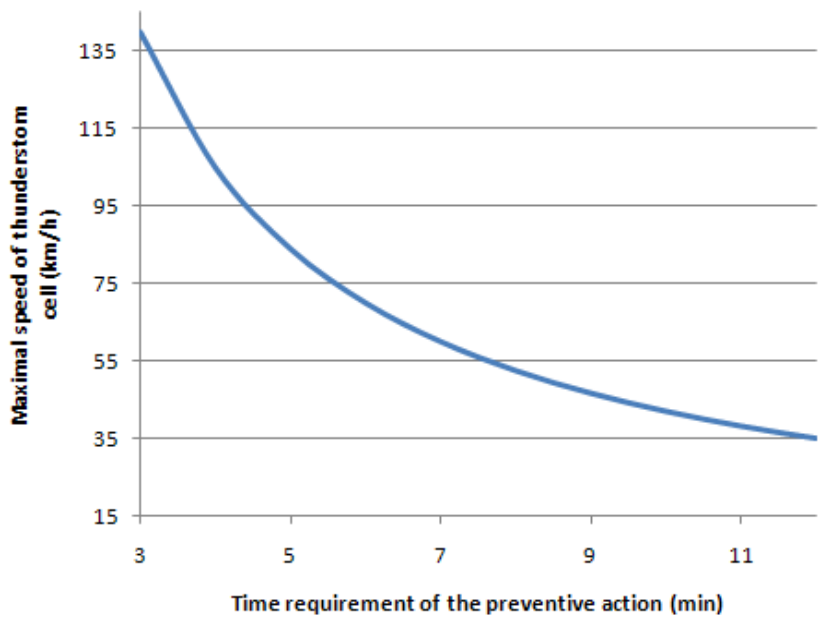

Fig. (11). Maximum speed of thunderstorm cell producing a timely alarm $v s$ time spent on executing the preventive action $(\mathrm{WZ}=12 \mathrm{~km}$; $\mathrm{DZ}=5 \mathrm{~km})$.

The time function of the speed of the thunderstorm cell is estimated using the simulated distance values of the sensor. At a given moment the actual $\mathrm{v}_{\text {storm }}$ value was calculated from the average of the previous 20 distance data. Despite of the averaging the deviation of velocity values remained quite high. This results in a similar tendency in $d_{\text {crit }}$ as it can be seen in Fig. (12) - having smaller $d_{c r i t}$ in case of cells with 
higher propagation speed. Then as the velocity of the cell decreases, the $d_{\text {crit }}$ increases again.

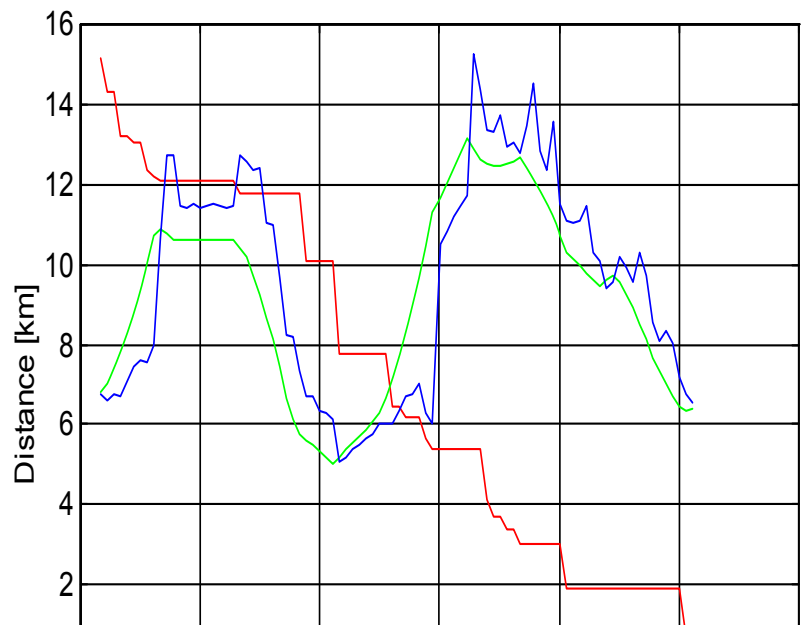

Fig. (12). Comparison of alarming. Red curve: actual distance between the closest lightning strike (determined by the detector) and the object to be protected. Blue curve: $d_{\text {crit }}$. Green curve: $d_{\text {crit }}$ modified by the FIS.

That leads to a difficult applicability for short $t_{a c t}$ values, regarding, that sudden changes in $d_{c r i t}$ cannot be followed, but using a FIS yields more accurate value. The use of the critical distance is similar in both HRPLP and FPLP. In this case the time requirement of preventive action was assumed to be 15 min (for both FPLP and HRPLP, also using such an action provides more spectacular results in this case). Estimation of $d_{c r i t}$ without FIS results in a late alarm. (Less than $15 \mathrm{~min}$. is the time difference between giving the alarm - the blue and the red curve cross - and the red curve crossing at $5 \mathrm{~km}$ value, see Fig. 12).

The fuzzy inference system modifies the value of $d_{\text {crit }}$ in such a way, that the previous deviations - the inaccuracy of the local detector - are reduced. Comparing the probability of late alarms to the calculated values of Fig. (12) it can be seen, that the application of dynamic $d_{c r i t}$ distances result in higher probabilities of late alarms for shorter $t_{a c t}$ values, but the application of fuzzy logic reduces it significantly.

Also the performance of HRPLP may be improved by data smoothing, just like in the case of ZPLP. Moreover the critical distance may be intentionally 'oversized' (by slightly overestimating cell speed) to provide more effective protection.

Oversizing of the critical distance in HRPLP is required to take into account the distortion which is caused by smoothing the data (using averaging techniques given by (4) or (5)) provided by the standalone sensor. Not oversizing the critical distance would result in late alarms in some cases, when the thunderstorm cell accelerates rapidly close to the critical distance. In these cases data smoothing would result in underestimating cell speed unless the critical distance is defined to be a bit bigger than what would be calculated considering the cell speed and the execution time only.

Again for both of these solutions the most important benchmark is their capability of giving alarms in time, or in other words, the probability of late alarms. Fig. (13) shows a comparison of HRPLP and FPLP using simulated sensor data as input. It is clearly seen that HRPLP is inferior if raw data is used without any pre-processing, but in case of advanced techniques its performance increases dramatically.

Note the difference between Fig. (13) and Fig. (10) - the case of ZPLP. The explanation for the different behaviour of these systems is that while in case of ZPLP there is a fixed time to execute the preventive action, HRPLP and FPLP 'adapt' this time to the approach of the thunderstorm cell. However if the execution time of the action is low, then rapid changes in the velocity of the thunderstorm cell may result in either HRPLP or FPLP 'allow' the thunderstorm cell come too close before giving the alarm.

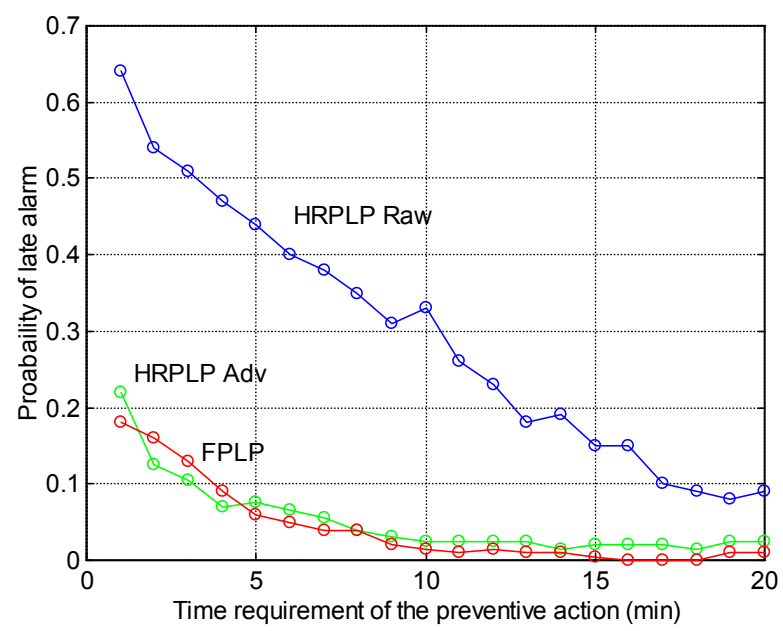

Fig. (13). Probability of late alarms in case of HRPLP and FPLP (red and blue curve respectively).

As Fig. (13) shows both methods are capable of providing adequate protection, but it will always come at a cost of unnecessary alarms.

\section{SUMMARY AND DISCUSSION}

We introduced the application of the local detectors in preventive lightning protection in this paper. PLP is new approach to lighting protection, as it incorporates the use of preventive actions instead of installing certain devices to the object to be protected. Since forecasting is a key element of PLP, the stand-alone lightning detectors may be used as a tool of detection and distance approximation.

First we have shown that the stand-alone devices may be used as a forecasting tool using statistical methods only. A brief description was given about the performance of the detectors and how its characteristics may be described.

Then we have introduced the ways of using the standalone devices in PLP. For each PLP type (ZPLP, HRPLP and FPLP) the application of the detectors was described (along with a short theoretical description). Also each PLP type was tested using a Monte Carlo simulation.

The simulations have shown that using statistical data the standalone detector performs well if the ZPLP solution is planned well. The probability of late alarms is very low (below 0.1) if the time requirement of a preventive action does not exceed 10 minutes for the given arrangement. 
When using FPLP or HRPLP even better results may be obtained, the probability of late alarms gets even lower. This is due to the more complex calculations done. However a weak spot of both FPLP and HRPLP was also shown, namely that in case of too short preventive actions, late alarms may occur.

Based on our results it was shown, that despite the inaccuracies stand-alone detectors can be used very effectively - both in terms of cost and protection - in PLP. Either by using ZPLP, HRPLP or FPLP, adequate protection can be realized if planning is done carefully.

\section{ACKNOWLEDGEMENT}

Declared none.

\section{CONFLICT OF INTEREST}

Declared none.

\section{REFERENCES}

[1] Gulyás A, Németh B, Szonda S, Berta I. Application of preventive measures in lightning protection; presented at the 28th International Conference on Lightning Protection ICLP; 2006; Kanazawa, Japan.

[2] Mäkelä JS, Porjo N, Jantunen J, et al. Single-station narrowband ranging of active storm cells without lightning-type discrimination. J Atmos Solar-Terrest Phys 2009; 71(8-9): 911-22.

[3] Mäkelä JS, Porjo N, Ahola T, Hämäläinen A, Jantunen J. Using full-flash narrowband energy for ranging of lightning ground strokes. J Atmos Solar-Terrest Phys 2008; 70(1): 156-68.

[4] Mäkelä JS. Electromagnetic signatures of lightning near the HF frequency band. Finnish Meteorological Institute Conttribuution. Academic Dissertation in Physics 2009.

[5] Cummins KL, Murphy MJ, Bardo EA, et al. A combined TOA/MDF technology upgrade of the U.S. National Lightning Detection Network. J Geophys Res 1998; 103(8): 9035-44.

[6] Beasley WH, Byerley LG, Swenson JA, Hinckley A, Bogoev IG. Development and applications of a new, low-power, lowmaintenance electric-field meter for research and hazard-warning decision support. presented at the 13th International Conference on Athmospheric Electricity. Beijing, China 2007.

[7] Montanya J, Bergas J, Hermoso B. Electric field measurements at ground level as a basis of lightning hazard warning. Presented at the 26th ICLP. Krakow 2002; p. 8b3.
[8] Beasley WH, Williams DE, Hyland PT. Analysis of surface electric-field contours in relation to cloud-to-ground lightning flashes in air-mass thunderstorms at the Kennedy Space Center. presented at the 20th International Lightning Detection Conference. Tucson AZ, USA 2008.

[9] Proctor DE. Lightning flashes with high origins. J Geophys Res 1997; 102 (2): 1693-706.

[10] Holle RL, Lopez RE, Zimmermann C. Updated recommendations for lightning safety 1998. Bull Am Meteorol Soc 1999; 80(10): 2035-41.

[11] Lojou JY, Murphy MJ, Demetriades NWS, Cummins KL. Assessment of thunderstorm lifecycle using total lightning systems; presented at the 13th International Conference on Athmospheric Electricity. Beijing, China 2007; pp. os8-6.

[12] Gulyás A, Németh B, Kiss I, Berta I. The theoretical framework of preventive lightning protection; presented at the 29th International Conference on Lightning Protection ICLP. Uppsala, Sweden 2008.

[13] Mendes J, Domingues MO. Lightning path simulation based on the stepped leader: electrical conductivity effects. J Atmos SolarTerrest Phys 2005; 67(14): 1287-97.

[14] Németh B, Gulyás A, Kiss I, Berta I. Efficiency of lightning detection systems in preventive lightning protection; presented at the International Youth Conference on Energetics. Budapest 2007.

[15] Gulyás A, Kiss I. High reliability preventive lightning protection; in 20th International Lightning Detection Conference. Tucson AZ, USA 2008; p. 13.

[16] Gorin BN, Kuzhekin IP, Sokolov KI, Simkin AV. System of a thunderstorm warning on the Ostankino TV-Tower; presented at the 26th International Conference on Lightning Protection. Krakow 2002.

[17] NASA facts: Lightning and the space program; 2006; FL, FS-200510-031-KSC (Rev. 2006); NASA, USA.

[18] Németh B, Gulyás A, Berta I. Increasing safety in live line maintenance applying preventive lightning protection; presented at the 13th International Conference on Athmospheric Electricity. Beijing, China 2007.

[19] Nemeth B, Gulyás A, Kiss I. Lightning protection of open air mass performances; presented at the 29th International Conference on Lightning Protection ICLP. Uppsala, Sweden 2008.

[20] Németh B, Kiss I. Application of fuzzy logic based expert system in preventive lightning protection. J Electrostat 2009; 67(2-3): 47781

[21] Van Broekhoven E, De Baets B. Fast and accurate center of gravity defuzzification of fuzzy system outputs defined on trapezoidal fuzzy partitions. Fuzzy Sets Syst 2006; 157(7): 904-18. 